\title{
Computerized Data Management for Almond Breeding Programs
}

\author{
F. Dicenta and J.E. Garcia \\ Centro de Edafología y Biología Aplicada del Segura (Consejo Superior \\ de Investigaciones Científicas), Apartado 4195, 30080 Murcia, Spain
}

Additional index words. data recording

We describe a totally computerized system that facilitates the recording, organizing, and storing of data gathered during a large almond breeding program. A portable PSION Organizer II (PSION PLC, London) microcomputer (CMOS 6303X microprocessor, 32 K RAM, 32 K ROM, 12 to 128 K EPROM) was used for entry of data directly in the field or in the laboratory. A COMMS-LINK (PSION PLC) software, written for the PSION, was used for interfacing the portable microcomputer to PC, running MS-DOS. PSION Organizer II uses programs written in the OPL language. This program will not run in other devices.

Twenty-three characteristics of 2518 seedling were tracked and evaluated according to international norms (Gulcan, 1985). To facilitate the recording of data, nine separate programs in OPL language were written, all displaying the number of the tree being examined and the characteristic being evaluated. The programs are:

1) Flowering: Displays the Julian day of the year and prompts the operator to record the initial day of flowering (IF). This part

Received for publication 8 Mar. 1991. Accepted for publication 10 Sept. 1991. The cost of publishing this paper was defrayed in part by the payment of page charges. Under postal regulations, this paper therefore must be hereby marked $a d$ vertisement solely to indicate this fact. could be modified if the IF occurred some days before or after the tree was examined and could even be erased from the screen if this datum were not needed; in the latter case, the program will prompt for the IF at the next data-recording session. If the IF was entered, the next time that the tree is visited, the program would prompt for the full flowering date $(\mathrm{FF})$. If FF was recorded, the program would ask for the flowering density. Once these data were entered, the next time the program would prompt for final flowering date. If this were entered, at the next visit the program would indicate that all the flowering data were previously recorded.

2) Production: Asks for data on the productivity, scored from 0 (nil) to 5 (maximum).

3) Maturation: Displays the Julian day of the year and prompts for the day of fruit ripening. As with the flowering program, this entry can be modified (when ripening occurs before or after the day in question) or erased from the screen (when no data will be recorded). Once data are recorded, the program will show this fact the next time the tree is visited.

4) and 5) Self-compatibility: These two programs record data concerning the percentage of self-compatibility. The former asks for the number of the bagged flowers on each tree, and the latter asks for the number of set fruits after 6 weeks.
6) Fruit: Asks for the number of the tree corresponding to the sample about to be analyzed. Once this number is entered, it permits the following data to be recorded: weight of nuts and kernels, softness of shell, number of empty shells, shape and thickness of kernel, rugosity, kernel color intensity, number of kernels with defects, type of defect, kernel flavor, and overall evaluation of the fruit. If the code number introduced corresponds to a tree whose fruit has already been examined, the program will indicate this fact and provide the possibility of modifying previously introduced data (error correction), erasing the same (in the case of a incorrectly numbered tree), or keeping the data unchanged, in which case the program will demand a new code number.

7) Tree: Permits one to enter data on the habit of the tree (extremely upright, upright, spreading, drooping, or weeping) and its ramification (sparse, intermediate, dense).

8) Vigor: Asks for the circumference of the tree trunk.

9) Floral bud localization: Permits one to enter the percentage of floral buds in three locations: one-year-old shoots, spurs, and mixed.

In addition, another program, Translator, written in Pascal, which runs on the PC, generates ASCII files to be used by programs in the minicomputer. This program permits modification of the files in the PC and transfers the data recorded by the PSION to a dBASE IV (Ashton-Tate, Torrance, Calif.).

An important improvement in the mechanization of recording and filing data has been outlined. This system could be applied, with slight modifications, to most fruit breeding programs.

All the software described above is available on request, by sending a diskette.

\section{Literature Cited}

Gulcan, R. 1985. Descriptor list for almond (Pru nus amygdalus) (revised). Intl. Board for Plant Genet. Resources, Rome. 